\title{
Restoration of plasma kidney and liver biomarkers in doxorubicin-treated Wistar rats by aqueous extracts of Pleurotus tuberregium sclerotia and Cnidoscolus aconitifolius leaves
}

\author{
Catherine C. IKeWUChi ${ }^{1}$, Jude C. IKeWUChi ${ }^{1}$, Mercy O. Ifeanacho ${ }^{2 *}$ \\ ${ }^{1}$ Department of Biochemistry, Faculty of Science, University of Port Harcourt, P.M.B. 5323, Port Harcourt, Nigeria \\ ${ }^{2}$ Department of Food, Nutrition and Home Science, Faculty of Agriculture, University of Port Harcourt, Nigeria
}

\begin{abstract}
The ability of aqueous extracts of sclerotia of Pleurotus tuberregium and leaves of Cnidoscolus aconitifolius to regulate plasma markers of kidney and liver function/integrity was investigated in doxorubicin-treated Wistar rats. Doxorubicin (dissolved in normal saline) was injected intraperitoneally $(15 \mathrm{mg} / \mathrm{kg}$ body weight) into the rats; metformin was daily administered orally at $250 \mathrm{mg} / \mathrm{kg}$, while the extracts were daily administered orally at doses of 50,75 , and $100 \mathrm{mg} / \mathrm{kg}$. Compared to the test control, in both the doxorubicin pre-treatment (or ameliorative) study and the extract pre-treatment (protective) studies, the extracts and metformin-treated groups had significantly lower $(P<0.05)$ plasma levels of alkaline phosphatase, alanine transaminase and aspartate transaminase, and concentrations of creatinine, urea, and blood urea nitrogen. However, the plasma globulin, albumin, and total protein concentrations and the albumin/globulin ratio of the extract and metformin-treated groups were significantly higher $(P<0.05)$. The extracts prevented (in the protective study) or attenuated (in the ameliorative study) doxorubicin-induced increase in the levels of plasma markers of kidney and liver function/integrity, and afforded protection or recovery towards near-normal values.
\end{abstract}

Key words: albumin/globulin ratio, alkaline phosphatase, blood urea nitrogen, creatinine, doxorubicin, transaminases

\section{Introduction}

Doxorubicin is a well-established and highly effective anti-neoplastic agent that is used to treat several adult and paediatric cancers such as solid tumours, leukaemia, lymphomas, and breast cancer (Carvalho et al., 2009; Octavia et al., 2012). The successful use of doxorubicin has been hampered by its toxicity to numerous organs such as the heart (Yilmaz et al., 2006; Carvalho et al., 2009; Rashid et al., 2013; Indu et al., 2014; Afsar et al., 2017; Bordbar et al., 2019 ; Ikewuchi et al., 2021a), liver (Carvalho et al., 2009; Indu et al., 2014; Chen et al., 2016; Alghorabi et al., 2019; Ikewuchi et al., 2021b), kidneys (Yilmaz et al., 2006; Carvalho et al., 2009; Rashid et al., 2013; Ren et al., 2016; Bordbar et al., 2019; Saba- pathy et al., 2019; Ikewuchi et al., 2021c), and lungs (Vapa et al., 2012; Jagetia and Lalrinpuii, 2018). Hepatonephrotoxicity induced by doxorubicin is accompanied by increased plasma levels of creatinine, urea, uric acid, gamma-glutamyl transferase, lactate dehydrogenase, alanine transaminase, aspartate transaminase, and alkaline phosphatase and decreased plasma albumin and total protein levels (Öz and İlhan, 2006; Yilmaz et al., 2006; Lee et al., 2013; Ahmed et al., 2019a).

Various bioactive compounds of plant origin have been reported to prevent or mitigate the nephrotoxicity and hepatotoxicity of doxorubicin (Surai, 2015). These compounds include allicin, caffeic acid, carotenoids (e.g. lycopene), catechin, ellagic acid, epicatechin, epigalloca-

\footnotetext{
* Corresponding author: Department of Food, Nutrition and Home Science, Faculty of Agriculture, University of Port Harcourt, Nigeria; e-mail: mifeanacho@yahoo.com
} 
techin gallate, naringenin, quercetin, and silymarin, all of which have been reported to exert hepato-nephroprotective effects through attenuation of doxorubicin-induced oxidative stress in the liver and kidneys (Yagmurca et al., 2004; Kalender et al., 2005; Yilmaz et al., 2006; Gokcimen et al., 2007; El-Shitany et al., 2008; Patel et al., 2010; Rašković et al., 2011; Indu et al., 2014; Jambhulkar et al., 2014; Rudolfová et al., 2014; Surai, 2015; Omar et al., 2016; Ahmed et al., 2019a,b).

Numerous studies have shown that the sclerotia of Pleurotus tuberregium and leaves of Cnidoscolus aconitifolius contain the above mentioned antioxidants, in addition to ascorbic and chlorogenic acids, and other antioxidants belonging to the following family of phytochemicals: simple and polyphenols, glycosides, phytosterols and saponins (Ijeh et al., 2009; Ikewuchi and Ikewuchi, 2009, 2011; Azeez et al., 2010; Ikewuchi et al., 2013a,b, 2014, 2017; Otitolaiye and Asokan, 2016; KuriGarcía et al., 2017; Ifeanacho et al., 2019a,b, 2020). Extracts of $P$. tuberregium sclerotia and $C$. aconitifolius leaves have been reported to protect the liver and kidney (Oyagbemi and Odetola, 2010; Saba et al., 2010; Adaramoye and Aluko, 2011; Adaramoye et al., 2011; Ikewuchi et al., 2017; Iwu et al., 2020). They have also been reported to be hypolipidaemic, anti-diabetic (Oladeinde et al., 2007; Ikewuchi et al., 2013a, 2021d; Achi et al., 2017; Ifeanacho et al., 2019a), anti-hypertensive (Ikewuchi et al., 2013a, 2014), and haematomodulatory (Azeez et al., 2010; Ifeanacho et al., 2020; Iwu et al., 2020; Onasanwo et al., 2020). In the present study, the ability of aqueous extracts of $P$. tuberregium sclerotia and $C$. aconitifolius leaves to regulate plasma markers of kidney and liver function/integrity was investigated in doxorubicin-treated rats.

\section{Materials and methods}

\section{Procurement of materials}

Fresh samples of the sclerotia of $P$. tuberregium were purchased from Mile 1 Market in Port Harcourt, Nigeria, while fresh leaves of $C$. aconitifolius (hospital too far) were collected from Farm Gardens in Aluu Community of Rivers State, Nigeria, and were duly identified as previously reported (Ikewuchi and Ikewuchi, 2009, 2011; Ikewuchi et al., 2013a,b, 2014, 2017; Ifeanacho et al., 2019a,b, 2020). Ninety Wistar rats (weight $80-130 \mathrm{~g}$ ) were obtained from the Animal House of the
Department of Physiology, University of Port Harcourt, Nigeria. The alanine transaminase, aspartate transaminase, alkaline phosphatase, creatinine, and urea kits were the products of Randox Laboratories Ltd., County Antrim, UK.

\section{Preparation of extracts}

The sclerotia and leaves were cleaned of dirt and dried before grinding into powder. The powders $(5.3 \mathrm{~kg}$ of $P$. tuberregium sclerotia and $5 \mathrm{~kg}$ of $C$. aconitifolius) were separately soaked in $10 \mathrm{~L}$ of hot (boiled) water for $12 \mathrm{~h}$. The resultant mixtures were filtered using a sieve cloth. The filtrates were then concentrated using a rotary evaporator prior to freeze-drying, yielding $145 \mathrm{~g}$ and $131 \mathrm{~g}$ of $P$. tuberregium sclerotia and $C$. aconitifolius leaves extracts, respectively. The resultant extracts of $P$. tuberregium sclerotia and $C$. aconitifolius leaves (hereafter termed PTSE and CALE, respectively) were weighed, reconstituted in distilled water, and administered to the experimental animals according to their individual weights and doses of their respective groups.

\section{Experimental design}

All experimental procedures in this study were performed in accordance with the ethical guidelines for investigations using laboratory animals and complied with the guide for the care and use of laboratory animals (National Research Council, 2011). The animals were weighed and sorted into 18 groups of five animals each, with an average difference of $\leq 3 \mathrm{~g}$ in mean weight (FAO, 1991). Of these 18 groups, nine groups were used for the ameliorative study, while the remaining nine groups were used for the protective study. They were housed in cages at the Department of Physiology, University of Port Harcourt and allowed access to water and feed $a d$ libitum. The treatment commenced after 1 week of acclimatization. The doxorubicin dose was adopted from Song et al. (2019). The doses of administration of the $P$. tuberregium sclerotia extract was adopted and modified from Ifeanacho et al. (2019a, 2020), that of $C$. aconitifolius extract was adopted and modified from Adaramoye and Aluko (2011), while that of metformin was adopted from Zilinyi et al. (2018).

\section{Ameliorative (or doxorubicin pre-treatment) study}

Doxorubicin was dissolved in normal saline and injected intraperitoneally ( $15 \mathrm{mg} / \mathrm{kg}$ body weight) into all 
rats from all the groups, except for normal control which was administered normal saline instead of doxorubicin solution. After 1 week, the treatment was commenced and lasted for 14 days. Diabetmin ${ }^{\mathrm{TM}}$ (metformin $\mathrm{HCl}$ ) was dissolved in distilled water and orally administered daily at the dose of $250 \mathrm{mg} / \mathrm{kg}$ to the metformin group. The extracts were administered through the same route at the dose of $50 \mathrm{mg} / \mathrm{kg}$ to PTSE- $50 \mathrm{mg}$ (P. tuberregium sclerotia extract) and CALE-50 $\mathrm{mg}$ (C. aconitifolius leafextract); $75 \mathrm{mg} / \mathrm{kg}$ to PTSE-75 $\mathrm{mg}$ (PTSE) and CALE- $75 \mathrm{mg}$ (CALE); and $100 \mathrm{mg} / \mathrm{kg}$ to PTSE-100 mg (PTSE) and CALE-100 mg (CALE). The normal and test control groups received distilled water instead of the extracts.

\section{Protective (or extract pre-treatment) study}

Diabetmin $^{\mathrm{TM}}$ (dissolved in distilled water) was orally administered daily at the dose of $250 \mathrm{mg} / \mathrm{kg}$ to the metformin group. The extracts were administered through the same route at the dose of $50 \mathrm{mg} / \mathrm{kg}$ to PTSE $-50 \mathrm{mg}$ (PTSE) and CALE-50 mg (CALE); $75 \mathrm{mg} / \mathrm{kg}$ to PTSE-75 mg (PTSE) and CALE-75 mg (CALE); and $100 \mathrm{mg} / \mathrm{kg}$ to PTSE-100 mg (PTSE) and CALE-100 mg (CALE). The normal and test control groups received distilled water instead. On day 12, doxorubicin (in normal saline) was intraperitoneally injected $(15 \mathrm{mg} / \mathrm{kg})$ into all the groups, except for normal control which was administered normal saline instead.

\section{Collection of plasma samples}

On day $14^{\text {th }}$ of the treatment in each study, the animals were sacrificed under chloroform anaesthesia, and blood samples were collected into heparin bottles. The blood samples were centrifuged at $1000 \mathrm{rpm}$ for $10 \mathrm{~min}$, and the plasma was collected into plain sample bottles and stored in a refrigerator for use in the assay.

\section{Determination of plasma markers of kidney and liver function/integrity}

The plasma levels of alanine transaminase (ALT), aspartate transaminase (AST), alkaline phosphatase (ALP), total protein, albumin, creatinine, and urea were assayed according to the kit manufacturer's instructions. The plasma globulin level and the plasma albumin/globulin ratio were calculated using the following formulae (Paul, 2013):

1) Plasma globulin concentration $=$

$=$ total protein concentration - albumin concentration
2) Plasma albumin to globulin ratio $=$

$$
=\frac{\text { albumin concentration }}{\text { globulin concentration }}
$$

The urea to creatinine ratio was calculated as follows (Manoeuvrier et al., 2017):

Urea $/$ creatinine ratio $=$

$$
=\frac{\text { Serum urea concentration }[\mathrm{mmol} / 1]}{\text { Serum creatinine concentration }[\mathrm{mmol} / 1]}
$$

\section{Determination of per cent recovery or protection}

The level of restoration or safeguard of kidney and liver function/integrity, denoted as per cent recovery/ protection, was calculated as follows (Ikewuchi et al., 2021b):

Per cent recopvery $($ or protection $)=$

$$
=\frac{\text { Parameter }_{\text {test control }}-\text { Parameter }_{\text {treatment }}}{\text { Parameter }_{\text {test control }}-\text { Parameter }_{\text {normal control }}} \times 100
$$

\section{Statistical analysis of data}

Statistical calculations were performed with the software Excel 2010 (Data Analysis Add-in). All data are expressed as mean \pm standard error of the mean (SEM) and were analysed using one-way analysis of variance. Significant difference of the mean values was determined using the least significant difference test, and $P<0.05$ was considered statistically significant.

\section{Results}

\section{Regulation of plasma markers of kidney function}

The influence of aqueous extracts of the sclerotia of $P$. tuberregium and the leaves of $C$. aconitifolius on plasma markers of kidney function in doxorubicin-treated rats is shown in Table 1. In both the doxorubicin pretreatment (ameliorative) study and the extract pre-treatment (protective) study, the plasma creatinine levels $(263.13 \pm 3.11$ and $172.00 \pm 4.18 \mu \mathrm{mol} / \mathrm{l}$, respectively) and urea levels $(14.11 \pm 0.41$ and $13.67 \pm 0.25 \mathrm{mmol} / \mathrm{l}$, respectively) of the test control group were significantly higher $(P<0.05)$ than those of all the other groups. The CALE-100 mg group had the least plasma creatinine levels $(19.85 \pm 2.09$ and $18.19 \pm 1.28 \mu \mathrm{mol} / \mathrm{l}$, respectively), while the least plasma urea concentrations were observed in the CALE-50 mg group ( $5.25 \pm 0.15 \mathrm{mmol} / \mathrm{l})$ of the ameliorative study and in the PTSE-75 $\mathrm{mg}$ group $(5.15 \pm 0.24 \mathrm{mmol} / \mathrm{l})$ of the protective study. In both the ameliorative and protective studies, the blood urea 
Table 1. Influence of aqueous extracts of Pleurotus tuberregium sclerotia and Cnidoscolus aconitifolius leaves on plasma markers of kidney function in doxorubicin-treated rats

\begin{tabular}{|c|c|c|c|c|c|c|c|c|}
\hline \multirow{2}{*}{ Treatment } & \multicolumn{2}{|c|}{ Creatinine $[\mu \mathrm{mol} / \mathrm{l}]$} & \multicolumn{2}{|c|}{ Urea [mmol/1] } & \multicolumn{2}{|c|}{ Blood urea nitrogen [mg/dl] } & \multicolumn{2}{|c|}{ Urea nitrogen/creatinine ratio* } \\
\hline & ameliorative & protective & ameliorative & protective & ameliorative & protective & ameliorative & protective \\
\hline Normal control & $30.43 \pm 1.62^{\mathrm{a}, \mathrm{g}}$ & $30.43 \pm 1.62^{\mathrm{a}}$ & $5.38 \pm 0.14^{\mathrm{a}}$ & $5.38 \pm 0.14^{\mathrm{a}}$ & $15.10 \pm 0.40^{\mathrm{a}}$ & $15.10 \pm 0.40^{\mathrm{a}}$ & $183.01 \pm 10.63^{\mathrm{a}, \mathrm{c}, \mathrm{d}}$ & $183.01 \pm 10.63^{\mathrm{a}}$ \\
\hline Test control & $263.13 \pm 3.11^{c}$ & $172.00 \pm 4.18^{\mathrm{c}}$ & $14.11 \pm 0.41^{\mathrm{c}}$ & $13.67 \pm 0.25^{\mathrm{c}}$ & $39.63 \pm 1.16^{\mathrm{c}}$ & $38.39 \pm 0.69^{c}$ & $53.63 \pm 1.54^{\mathrm{c}}$ & $79.74 \pm 2.67^{\mathrm{c}, \mathrm{d}}$ \\
\hline Metformin & $50.72 \pm 2.42^{\mathrm{d}}$ & $39.69 \pm 2.09^{d}$ & $7.83 \pm 0.17^{\mathrm{d}}$ & $8.13 \pm 0.19^{d}$ & $21.99 \pm 0.49^{\mathrm{d}}$ & $22.83 \pm 0.55^{\mathrm{d}}$ & $157.43 \pm 9.87^{\mathrm{a}, \mathrm{c}, \mathrm{d}}$ & $206.37 \pm 6.42^{\mathrm{a}}$ \\
\hline PTSE-50 mg & $116.87 \pm 1.21^{\mathrm{e}}$ & $96.59 \pm 3.37^{\mathrm{e}}$ & $8.99 \pm 0.46^{\mathrm{e}}$ & $9.71 \pm 0.17^{\mathrm{b}}$ & $25.25 \pm 1.29^{\mathrm{e}}$ & $27.28 \pm 0.49^{b}$ & $607.26 \pm 325.73^{b}$ & $100.86 \pm 2.41^{\mathrm{d}}$ \\
\hline PTSE-75 mg & $24.81 \pm 1.28^{\mathrm{f}, \mathrm{g}}$ & $112.46 \pm 2.09^{\mathrm{f}}$ & $11.22 \pm 0.32^{\mathrm{f}}$ & $5.15 \pm 0.24^{\mathrm{a}}$ & $31.52 \pm 0.89^{b}$ & $14.46 \pm 0.67^{\mathrm{a}}$ & $455.88 \pm 14.93^{\mathrm{b}, \mathrm{d}}$ & $45.80 \pm 2.04^{\mathrm{c}}$ \\
\hline PTSE-100 mg & $233.74 \pm 4.83^{\mathrm{b}}$ & $100.89 \pm 4.38^{\mathrm{e}}$ & $7.72 \pm 0.16^{\mathrm{d}}$ & $10.31 \pm 0.38^{b}$ & $21.68 \pm 0.46^{\mathrm{d}}$ & $28.97 \pm 1.06^{\mathrm{b}}$ & $33.14 \pm 1.11^{\mathrm{a}, \mathrm{c}}$ & $102.61 \pm 3.12^{d}$ \\
\hline CALE-50 mg & $36.39 \pm 1.48^{\mathrm{a}}$ & $124.04 \pm 5.28^{g}$ & $5.25 \pm 0.15^{\mathrm{a}, \mathrm{b}}$ & $9.70 \pm 0.30^{b}$ & $14.75 \pm 0.41^{\mathrm{a}}$ & $27.25 \pm 0.83^{b}$ & $144.87 \pm 3.20^{\mathrm{a}, \mathrm{c}, \mathrm{d}}$ & $78.85 \pm 4.30^{\mathrm{c}, \mathrm{d}}$ \\
\hline CALE-75 mg & $34.73 \pm 2.45^{\mathrm{a}}$ & $62.85 \pm 1.48^{\mathrm{h}}$ & $7.89 \pm 0.25^{\mathrm{d}}$ & $5.56 \pm 0.11^{\mathrm{a}}$ & $22.16 \pm 0.69^{\mathrm{d}}$ & $15.60 \pm 0.32^{\mathrm{a}}$ & $234.90 \pm 20.10^{\mathrm{a}, \mathrm{c}, \mathrm{d}}$ & $88.68 \pm 3.04^{\mathrm{c,d}}$ \\
\hline CALE-100 mg & $19.85 \pm 2.09^{\mathrm{f}}$ & $18.19 \pm 1.28^{b}$ & $5.32 \pm 0.19^{\mathrm{a}, \mathrm{b}}$ & $8.21 \pm 0.24^{\mathrm{d}}$ & $14.95 \pm 0.53^{\mathrm{a}}$ & $23.06 \pm 0.67^{d}$ & $290.12 \pm 42.59^{\mathrm{a}, \mathrm{c}, \mathrm{d}}$ & $470.43 \pm 54.86^{b}$ \\
\hline
\end{tabular}

Values are expressed as mean $\pm \mathrm{SEM}, n=5$ animals per group; values in the same column with different superscript letters differ significantly at $P<0.05$; ${ }^{*}$ has no unit

Table 2. Effects of aqueous extracts of Pleurotus tuberregium sclerotia and Cnidoscolus aconitifolius leaves on the concentrations (g/l) of plasma non-enzyme markers of liver function in doxorubicin-treated rats

\begin{tabular}{|c|c|c|c|c|c|c|c|c|}
\hline \multirow{2}{*}{ Treatment } & \multicolumn{2}{|c|}{ Albumin } & \multicolumn{2}{|c|}{ Total protein } & \multicolumn{2}{|c|}{ Globulin } & \multicolumn{2}{|c|}{ Albumin/globulin ratio* } \\
\hline & ameliorative & protective & ameliorative & protective & ameliorative & protective & ameliorative & protective \\
\hline Normal control & $12.354 \pm 0.495^{\mathrm{a}, \mathrm{d}}$ & $12.354 \pm 0.495^{\mathrm{a}, \mathrm{c}}$ & $142.937 \pm 5.988^{\mathrm{a}, \mathrm{e}}$ & $142.937 \pm 2.344^{\mathrm{a}}$ & $130.999 \pm 1.931^{\mathrm{a}, \mathrm{e}}$ & $130.999 \pm 1.931^{\mathrm{a}}$ & $0.094 \pm 0.003^{\mathrm{a}, \mathrm{b}}$ & $0.094 \pm 0.003^{\mathrm{a}}$ \\
\hline Test control & $7.172 \pm 0.412^{c}$ & $7.721 \pm 0.322^{b}$ & $101.443 \pm 2.374^{\mathrm{c}}$ & $117.423 \pm 0.794^{c}$ & $90.922 \pm 0.802^{c}$ & $109.701 \pm 0.891^{\mathrm{c}}$ & $0.079 \pm 0.005^{\mathrm{a}, \mathrm{e}}$ & $0.070 \pm 0.003^{b}$ \\
\hline Metformin & $10.410 \pm 0.055^{\mathrm{b}}$ & $12.631 \pm 0.912^{\mathrm{c}, \mathrm{d}}$ & $134.335 \pm 2.123^{\mathrm{a}, \mathrm{b}}$ & $135.906 \pm 2.980^{\mathrm{d}}$ & $123.925 \pm 2.269^{\mathrm{d}, \mathrm{e}}$ & $123.856 \pm 2.439^{\mathrm{d}}$ & $0.084 \pm 0.002^{\mathrm{a}, \mathrm{b}, \mathrm{d}}$ & $0.096 \pm 0.004^{\mathrm{a}}$ \\
\hline PTSE-50 mg & $10.974 \pm 0.312^{\mathrm{a}, \mathrm{b}}$ & $11.486 \pm 0.866^{\mathrm{a}, \mathrm{d}}$ & $129.986 \pm 4.311^{\mathrm{b}}$ & $134.939 \pm 0.995^{d}$ & $117.441 \pm 4.027^{\mathrm{b}, \mathrm{d}}$ & $123.453 \pm 1.583^{d}$ & $0.094 \pm 0.004^{\mathrm{a}, \mathrm{b}}$ & $0.093 \pm 0.008^{\mathrm{a}}$ \\
\hline PTSE-75 mg & $11.104 \pm 0.305^{\mathrm{a}, \mathrm{b}}$ & $10.272 \pm 0.877^{\mathrm{a}}$ & $122.617 \pm 6.496^{\mathrm{b}}$ & $128.376 \pm 2.216^{\mathrm{b}}$ & $113.566 \pm 6.305^{b}$ & $115.076 \pm 2.515^{\mathrm{b}, \mathrm{c}}$ & $0.100 \pm 0.006^{\mathrm{b}, \mathrm{c}}$ & $0.089 \pm 0.007^{\mathrm{a}, \mathrm{d}}$ \\
\hline PTSE-100 mg & $13.418 \pm 0.607^{\mathrm{d}}$ & $14.314 \pm 0.260^{\mathrm{c}}$ & $129.986 \pm 1.722^{\mathrm{b}}$ & $127.208 \pm 1.861^{\mathrm{b}}$ & $116.568 \pm 1.909^{\mathrm{b}, \mathrm{d}}$ & $112.893 \pm 1.683^{\mathrm{b}, \mathrm{c}}$ & $0.116 \pm 0.006^{\mathrm{c}}$ & $0.126 \pm 0.002^{c}$ \\
\hline CALE-50 mg & $10.410 \pm 0.239^{b}$ & $12.215 \pm 1.421^{\mathrm{a}, \mathrm{c}}$ & $153.302 \pm 6.340^{\mathrm{d}, \mathrm{e}}$ & $144.676 \pm 1.065^{\mathrm{a}}$ & $134.717 \pm 0.525^{\mathrm{a}}$ & $132.461 \pm 1.249^{\mathrm{a}}$ & $0.077 \pm 0.002^{\mathrm{a}, \mathrm{d}}$ & $0.093 \pm 0.011^{\mathrm{a}}$ \\
\hline CALE-75 mg & $11.972 \pm 1.660^{\mathrm{a}, \mathrm{b}, \mathrm{d}}$ & $10.688 \pm 0.531^{\mathrm{a}, \mathrm{d}}$ & $133.369 \pm 4.835^{\mathrm{a}, \mathrm{b}}$ & $128.053 \pm 2.607^{\mathrm{b}}$ & $121.397 \pm 4.876^{\mathrm{b}, \mathrm{d}, \mathrm{e}}$ & $118.653 \pm 3.785^{\mathrm{b}, \mathrm{d}}$ & $0.100 \pm 0.015^{\mathrm{b}, \mathrm{c}}$ & $0.088 \pm 0.006^{\mathrm{a}}$ \\
\hline CALE-100 mg & $11.235 \pm 0.457^{\mathrm{a}, \mathrm{b}}$ & $11.104 \pm 0.494^{\mathrm{a}, \mathrm{d}}$ & $161.718 \pm 4.740^{\mathrm{d}}$ & $163.651 \pm 2.086^{\mathrm{e}}$ & $150.483 \pm 4.692^{\mathrm{f}}$ & $152.546 \pm 1.960^{\mathrm{e}}$ & $0.075 \pm 0.004^{\mathrm{d}, \mathrm{e}}$ & $0.073 \pm 0.003^{\mathrm{b}, \mathrm{d}}$ \\
\hline
\end{tabular}

Values are expressed as mean $\pm \mathrm{SEM}, n=5$ animals per group; values in the same column with different superscript letters differ significantly at $P<0.05$; * has no unit 
Table 3. Ameliorative and protective effects of the extracts on the levels (U/l) of plasma enzyme markers of liver integrity

\begin{tabular}{l|c|c|c|c|c|c}
\hline \multirow{2}{*}{ Treatment } & \multicolumn{2}{|c|}{ Alkaline phosphatase } & \multicolumn{2}{c|}{ Aspartate transaminase } & \multicolumn{2}{c}{ Alanine transaminase } \\
\cline { 2 - 7 } & ameliorative & protective & ameliorative & protective & ameliorative & protective \\
\hline Normal control & $72.3 \pm 6.7^{\mathrm{a}, \mathrm{f}}$ & $72.3 \pm 6.7^{\mathrm{a}}$ & $328.4 \pm 13.9^{\mathrm{a}}$ & $328.4 \pm 13.9^{\mathrm{a}}$ & $151.0 \pm 5.3^{\mathrm{a}}$ & $151.0 \pm 5.3^{\mathrm{a}}$ \\
\hline Test control & $134.6 \pm 8.6^{\mathrm{c}}$ & $118.2 \pm 4.6^{\mathrm{c}}$ & $504.3 \pm 17.6^{\mathrm{c}}$ & $752.5 \pm 15.0^{\mathrm{c}}$ & $306.0 \pm 8.1^{\mathrm{c}}$ & $327.6 \pm 12.6^{\mathrm{c}}$ \\
\hline Metformin & $100.0 \pm 3.5^{\mathrm{d}}$ & $87.4 \pm 5.0^{\mathrm{d}, \mathrm{e}}$ & $422.0 \pm 16.6^{\mathrm{b}}$ & $305.0 \pm 4.8^{\mathrm{a}}$ & $155.7 \pm 3.8^{\mathrm{a}}$ & $151.0 \pm 6.7^{\mathrm{a}}$ \\
\hline PTSE-50 mg & $49.7 \pm 7.6^{\mathrm{e}}$ & $95.5 \pm 4.8^{\mathrm{e}}$ & $391.2 \pm 7.0^{\mathrm{b}}$ & $434.4 \pm 13.2^{\mathrm{d}}$ & $163.4 \pm 18.0^{\mathrm{a}}$ & $136.3^{\mathrm{a}} 5.7^{\mathrm{a}}$ \\
\hline PTSE-75 mg & $83.3 \pm 3.9^{\mathrm{a}, \mathrm{b}}$ & $33.9 \pm 0.9^{\mathrm{f}}$ & $406.5 \pm 8.9^{\mathrm{b}}$ & $334.4 \pm 13.8^{\mathrm{a}}$ & $206.9 \pm 6.4^{\mathrm{b}}$ & $216.0 \pm 6.8^{\mathrm{d}}$ \\
\hline PTSE-100 mg & $119.9 \pm 5.3^{\mathrm{c}}$ & $69.2 \pm 3.4^{\mathrm{a}, \mathrm{g}}$ & $406.7 \pm 10.2^{\mathrm{b}}$ & $385.0 \pm 7.1^{\mathrm{b}}$ & $189.9 \pm 4.8^{\mathrm{b}}$ & $145.6 \pm 5.6^{\mathrm{a}}$ \\
\hline CALE-50 mg & $87.6 \pm 4.2^{\mathrm{b}, \mathrm{d}, \mathrm{f}}$ & $76.1 \pm 3.7^{\mathrm{a}, \mathrm{d}}$ & $323.0 \pm 9.0^{\mathrm{a}}$ & $329.6 \pm 7.7^{\mathrm{a}}$ & $204.8 \pm 9.2^{\mathrm{b}}$ & $179.2 \pm 7.8^{\mathrm{b}}$ \\
\hline CALE-75 mg & $94.8 \pm 4.4^{\mathrm{b}, \mathrm{d}}$ & $50.4 \pm 2.1^{\mathrm{b}}$ & $414.0 \pm 3.0^{\mathrm{b}}$ & $406.7 \pm 8.1^{\mathrm{d}}$ & $115.8 \pm 4.7^{\mathrm{d}}$ & $142.1 \pm 6.9^{\mathrm{a}}$ \\
\hline CALE-100 mg & $71.1 \pm 3.3^{\mathrm{a}}$ & $60.3 \pm 1.9^{\mathrm{b}, \mathrm{g}}$ & $478.0 \pm 7.3^{\mathrm{c}}$ & $372.0 \pm 7.6 \mathrm{~b}$ & $206.4 \pm 5.8^{\mathrm{b}}$ & $172.8 \pm 4.1^{\mathrm{b}}$ \\
\hline
\end{tabular}

Values are expressed as mean \pm standard error of the mean, $n=5$ animals per group; values in the same column with different superscript letters differ significantly at $P<0.05$

nitrogen concentrations of the test control groups $(39.63 \pm 1.16$ and $38.39 \pm 0.69 \mathrm{mg} / \mathrm{dl}$, respectively) were significantly higher $(P<0.05)$ than those of all the other groups, with the CALE-100 mg group having the least value $(14.95 \pm 0.53 \mathrm{mg} / \mathrm{dl})$ in the ameliorative study and the PTSE-75 mg group having the least value (14.46 \pm $\pm 0.67 \mathrm{mg} / \mathrm{dl}$ ) in the protective study. In the ameliorative study, the urea nitrogen/ creatinine ratio of the test control group $(53.63 \pm 1.54)$ was significantly lower $(P<0.05)$ than those of the PTSE-50 $\mathrm{mg}$ group $(607.26 \pm 325.73)$ and the PTSE-75 mg group $(455.88 \pm$ \pm 14.93 ), but not significantly different from those of all the other groups. In the protective study, the urea nitrogen/creatinine ratio of the test control group $(79.74 \pm 2.67)$ was significantly lower $(P<0.05)$ than those of the normal control $(183.01 \pm 10.63)$, metformin (206.37 \pm 6.42$)$, and CALE-100 $\mathrm{mg}(470.43 \pm 54.86)$ groups, but not significantly different from those of all the other groups.

\section{Regulation of plasma markers of liver function/integrity}

As shown in Table 2, in the ameliorative and protective studies, the plasma albumin $(7.172 \pm 0.412$ and $7.721 \pm 0.322 \mathrm{~g} / \mathrm{l}$, respectively) and total protein (101.443 \pm 2.374 and $117.423 \pm 0.794 \mathrm{~g} / \mathrm{l}$, respectively) concentrations of the test control group were significantly $(P<0.05)$ lower than those of all the other groups. The lowest plasma albumin concentrations were observed in the PTSE $-100 \mathrm{mg}$ group $(13.418 \pm 0.607$ and $14.314 \pm$ $\pm 0.260 \mathrm{~g} / \mathrm{l}$, respectively), while the lowest total protein concentration was noted in the CALE-100 mg group (161.718 \pm 4.740 and $163.651 \pm 2.086 \mathrm{~g} / \mathrm{l}$, respectively). The plasma globulin concentration of the test control group $(90.922 \pm 0.802$ and $109.701 \pm 0.891 \mathrm{~g} / 1$, respectively) was significantly $(P<0.05)$ lower than those of all the other groups in the ameliorative study, while in the protective study, it was significantly $(P<0.05)$ lower than those of all the other groups, except PTSE-75 mg and PTSE-100 mg. The CALE-100 mg group had the highest globulin concentrations $(150.483 \pm 4.692$ and $152.546 \pm 1.960 \mathrm{~g} / \mathrm{l})$ in both studies. The plasma albu$\mathrm{min} /$ globulin ratio of the test control group $(0.079 \pm$ $\pm 0.005)$ in the ameliorative study was significantly lower $(P<0.05)$ than those of the PTSE-75 mg, PTSE-100 mg $(0.116 \pm 0.006$, the highest), and CALE-75 mg groups, but not significantly different from those of all the other groups. In the protective study, the plasma albumin/ globulin ratio of the test control group $(0.070 \pm 0.003)$ was significantly lower $(P<0.05)$ than those of all the other groups, except for the CALE-100 mg group, with the PTSE-100 mg group showing the highest value of $0.126 \pm 0.002$.

The plasma ALP levels of the test control group in both the ameliorative $(134.6 \pm 8.6 \mathrm{U} / \mathrm{l})$ and protective $(118.2 \pm 4.6 \mathrm{U} / \mathrm{l})$ studies (Table 3$)$ were significantly $(P<0.05)$ higher than those of the other groups, except for the PTSE-100 mg group (protective study only). The PTSE-50 mg group had the least plasma ALP level 
Table 4. Per cent recovery of plasma markers of liver and kidney integrity/function

\begin{tabular}{|c|c|c|c|c|c|c|c|}
\hline Parameter & Metformin & PTSE-50 mg & PTSE-75 mg & PTSE-100 mg & CALE-50 mg & CALE-75 mg & $\begin{array}{c}\text { CALE-100 } \\
\text { mg }\end{array}$ \\
\hline Albumin & $62.5 \pm 1.1^{\mathrm{a}}$ & $73.4 \pm 6.0^{\mathrm{a}}$ & $75.9 \pm 5.9^{\mathrm{a}}$ & $120.5 \pm 11.7^{b}$ & $62.5 \pm 4.6^{\mathrm{a}}$ & $92.6 \pm 32.0^{\mathrm{a}, \mathrm{b}}$ & $78.4 \pm 8.8^{\mathrm{a}}$ \\
\hline Total protein & $79.4 \pm 5.1^{\mathrm{a}}$ & $69.0 \pm 10.3^{\mathrm{a}}$ & $51.4 \pm 15.5^{\mathrm{a}}$ & $69.0 \pm 4.1^{\mathrm{a}}$ & $124.8 \pm 15.2^{b}$ & $77.1 \pm 11.6^{\mathrm{a}}$ & $144.9 \pm 11.3^{b}$ \\
\hline Globulin & $82.3 \pm 5.4^{\mathrm{c}, \mathrm{d}}$ & $66.2 \pm 10.0^{\mathrm{a}, \mathrm{d}}$ & $56.5 \pm 15.9^{\mathrm{a}}$ & $64.0 \pm 4.8^{\mathrm{a}, \mathrm{d}}$ & $109.3 \pm 1.3^{c}$ & $76.0 \pm 12.2^{\mathrm{a}, \mathrm{d}}$ & $148.6 \pm 11.7^{\mathrm{b}}$ \\
\hline $\begin{array}{l}\text { Albumin/ } \\
\text { globulin ratio }\end{array}$ & $12.5^{\mathrm{a}}$ & $102.9 \pm 30.3^{\mathrm{a}}$ & $142.0 \pm 40.2^{\mathrm{b}}$ & 252. & $-12.8 \pm 14.2^{\mathrm{a}}$ & $142 . £$ & $24.3^{\mathrm{a}}$ \\
\hline ALP & $55.6 \pm 5.7^{\mathrm{a}}$ & $136.4 \pm 12.2^{c}$ & $82.4 \pm 6.3^{\mathrm{b}, \mathrm{e}}$ & $23.5 \pm 8.5^{\mathrm{d}}$ & $75.4 \pm 6.7^{\mathrm{a}, \mathrm{b}}$ & $63.9 \pm 7.1^{\mathrm{a}, \mathrm{b}}$ & $102.0 \pm 5.2^{\mathrm{e}}$ \\
\hline AST & $46.8 \pm 9.5^{\mathrm{a}}$ & $64.3 \pm 4.0^{c}$ & $55.6 \pm 5.1^{\mathrm{a}, \mathrm{c}}$ & $55.5 \pm 5.8^{\mathrm{a}, \mathrm{c}}$ & $103.1 \pm 5.1^{b}$ & $51.3 \pm 1.7^{\mathrm{a}, \mathrm{c}}$ & $14.9 \pm 4.2^{\mathrm{d}}$ \\
\hline ALT & $97.0 \pm 2.5^{\mathrm{a}}$ & $92.0 \pm 11.6^{\mathrm{a}}$ & $63.9 \pm 4.1^{b}$ & $74.9 \pm 3.1^{b}$ & $65.3 \pm 5.9^{b}$ & $122.7 \pm 3.0^{c}$ & $64.3 \pm 3.7^{\mathrm{b}}$ \\
\hline Creatinine & $91.3 \pm 1.0^{\mathrm{a}}$ & $62.9 \pm 0.5^{c}$ & $102.4 \pm 0.6^{\mathrm{d}}$ & $12.6 \pm 2.1^{\mathrm{e}}$ & $97.4 \pm 0.6^{b}$ & $98.2 \pm 1.1^{b}$ & $104.5 \pm 0.9^{\mathrm{d}}$ \\
\hline Urea & $71.9 \pm 2.0^{\mathrm{a}}$ & $58.6 \pm 5.3^{c}$ & $33.1 \pm 3.6^{d}$ & $73.2 \pm 1.9^{\mathrm{a}}$ & $101.4 \pm 1.7^{\mathrm{b}}$ & $71.2 \pm 2.8^{\mathrm{a}}$ & $100.6 \pm 2.2^{b}$ \\
\hline BUN & $71.9 \pm 2.0^{\mathrm{a}}$ & $58.6 \pm 5.3^{c}$ & $33.1 \pm 3.6^{\mathrm{d}}$ & $73.2 \pm 1.9^{\mathrm{a}}$ & $101.4 \pm 1.7^{\mathrm{b}}$ & $71.2 \pm 2.8^{\mathrm{a}}$ & $100.6 \pm 2.2^{b}$ \\
\hline $\mathrm{Ur} / \mathrm{Cr}$ & $80.2 \pm 7.6^{\mathrm{a}, \mathrm{b}}$ & $427.9 \pm 251.8$ & $310.9 \pm 11.5^{\mathrm{b}, \mathrm{c}}$ & $-15.8 \pm 0.9^{\mathrm{a}}$ & $70.5 \pm 2.5^{\mathrm{a}, \mathrm{b}}$ & $140.1 \pm 15.5^{\mathrm{a}, \mathrm{b}}$ & $182.8 \pm 32.9^{\mathrm{a}, \mathrm{c}}$ \\
\hline
\end{tabular}

Values are expressed as mean \pm SEM, $n=5$ animals per group; values in the same row with different superscript letters differ significantly at $P<0.05$; BUN - blood urea nitrogen; $\mathrm{Ur} / \mathrm{Cr}$ - urea nitrogen/creatinine ratio

Table 5. Per cent protection of plasma markers of liver and kidney function/integrity

\begin{tabular}{l|c|c|c|c|c|c|c}
\hline \multicolumn{1}{c|}{ Parameter } & Metformin & PTSE-50 mg & PTSE-75 mg & $\begin{array}{c}\text { PTSE-100 } \\
\text { mg }\end{array}$ & CALE-50 mg & CALE-75 mg & $\begin{array}{c}\text { CALE-100 } \\
\text { mg }\end{array}$ \\
\hline Albumin & $106.0 \pm 19.7^{\mathrm{a}, \mathrm{b}}$ & $81.3 \pm 18.7^{\mathrm{a}}$ & $55.1 \pm 18.9^{\mathrm{a}}$ & $142.3 \pm 5.6^{\mathrm{b}}$ & $97.0 \pm 30.7^{\mathrm{a}, \mathrm{b}}$ & $64.0^{\mathrm{a}} \pm 11.5^{\mathrm{a}}$ & $73.0 \pm 10.7^{\mathrm{a}}$ \\
\hline Total protein & $72.4 \pm 11.7^{\mathrm{a}}$ & $68.7 \pm 3.9^{\mathrm{a}}$ & $42.9 \pm 8.7^{\mathrm{b}}$ & $38.4 \pm 7.3^{\mathrm{b}}$ & $106.8 \pm 4.2^{\mathrm{c}}$ & $41.7 \pm 10.2^{\mathrm{b}}$ & $181.2 \pm 8.2^{\mathrm{e}}$ \\
\hline Globulin & $66.5 \pm 11.5^{\mathrm{a}}$ & $64.6 \pm 7.4^{\mathrm{a}}$ & $25.2 \pm 11.8^{\mathrm{b}}$ & $15.0 \pm 7.9^{\mathrm{b}}$ & $106.9 \pm 5.9^{\mathrm{c}}$ & $42.0 \pm 17.7^{\mathrm{a}, \mathrm{b}}$ & $201.2 \pm 9.2^{\mathrm{d}}$ \\
\hline $\begin{array}{l}\text { Albumin/ } \\
\text { globulin ratio }\end{array}$ & $111.1 \pm 15.4^{\mathrm{a}}$ & $99.1 \pm 34.4^{\mathrm{a}}$ & $80.6 \pm 30.5^{\mathrm{a}, \mathrm{c}}$ & $241.0 \pm 7.4^{\mathrm{b}}$ & $95.3 \pm 48.9^{\mathrm{a}}$ & $77.0 \pm 26.3^{\mathrm{a}}$ & $10.3 \pm 13.8^{\mathrm{c}}$ \\
\hline ALP & $67.1 \pm 10.8^{\mathrm{a}}$ & $49.6 \pm 10.4^{\mathrm{a}}$ & $183.8 \pm 2.0^{\mathrm{c}}$ & $106.7 \pm 7.4^{\mathrm{d}, \mathrm{e}}$ & $91.7 \pm 8.0^{\mathrm{e}}$ & $147.7 \pm 4.5^{\mathrm{b}}$ & $126.3 \pm 4.0^{\mathrm{b}, \mathrm{d}}$ \\
\hline AST & $105.5 \pm 1.1^{\mathrm{a}}$ & $75.0 \pm 3.1^{\mathrm{c}}$ & $98.6 \pm 3.2^{\mathrm{d}}$ & $86.7 \pm 1.7^{\mathrm{b}, \mathrm{f}}$ & $99.7 \pm 1.8^{\mathrm{a}, \mathrm{b}, \mathrm{d}}$ & $81.5 \pm 1.9^{\mathrm{e}}$ & $89.7 \pm 1.8^{\mathrm{f}}$ \\
\hline ALT & $100.0 \pm 3.8^{\mathrm{a}}$ & $108.3 \pm 3.2^{\mathrm{a}}$ & $63.2 \pm 3.8^{\mathrm{c}}$ & $103.1 \pm 3.2^{\mathrm{a}}$ & $84.1 \pm 4.4^{\mathrm{b}}$ & $105.1 \pm 3.9^{\mathrm{a}}$ & $87.7 \pm 2.3^{\mathrm{b}}$ \\
\hline Creatinine & $93.5 \pm 1.5^{\mathrm{a}}$ & $53.3 \pm 2.4^{\mathrm{c}}$ & $42.1 \pm 1.5^{\mathrm{d}}$ & $50.2 \pm 3.1^{\mathrm{c}}$ & $33.9 \pm 3.7^{\mathrm{e}}$ & $77.1 \pm 1.0^{\mathrm{f}}$ & $108.6 \pm 0.9^{\mathrm{b}}$ \\
\hline BUN & $66.8 \pm 2.3^{\mathrm{a}}$ & $47.7 \pm 2.1^{\mathrm{c}}$ & $102.8 \pm 2.9^{\mathrm{b}}$ & $40.5 \pm 4.5^{\mathrm{c}}$ & $47.8 \pm 3.6^{\mathrm{c}}$ & $97.8 \pm 1.4^{\mathrm{b}}$ & $65.8 \pm 2.9^{\mathrm{a}}$ \\
\hline Ur/Cr & $66.8 \pm 2.3^{\mathrm{a}}$ & $47.7 \pm 2.1^{\mathrm{c}}$ & $102.8 \pm 2.9^{\mathrm{b}}$ & $40.5 \pm 4.5^{\mathrm{c}}$ & $47.8 \pm 3.6^{\mathrm{c}}$ & $97.8 \pm 1.4^{\mathrm{b}}$ & $65.8 \pm 2.9^{\mathrm{a}}$ \\
\hline
\end{tabular}

Values are expressed as mean \pm SEM, $n=5$ animals per group; values in the same row with different superscript letters differ significantly at $P<0.05 ; \mathrm{BUN}$ - blood urea nitrogen; Ur/Cr - urea nitrogen/creatinine ratio

$(49.7 \pm 7.6 \mathrm{U} / \mathrm{l})$ in the ameliorative study, while the PTSE-75 mg group had the least value $(33.9 \pm 0.9 \mathrm{U} / \mathrm{l})$ in the protective study. The plasma AST levels of the test control group in both the ameliorative (504.3 \pm $\pm 17.6 \mathrm{U} / \mathrm{l})$ and protective $(752.5 \pm 15.0 \mathrm{U} / \mathrm{l})$ studies were significantly higher $(P<0.05)$ than those of the other groups, except for the CALE-100 mg group (protective study). The CALE-50 mg group had the lowest plasma
AST level $(323.0 \pm 9.0 \mathrm{U} / \mathrm{l})$ in the ameliorative study, while the metformin group had the lowest level (305.0 \pm $\pm 4.8 \mathrm{U} / \mathrm{l})$ in the protective study. In both the ameliorative $(306.0 \pm 8.1 \mathrm{U} / \mathrm{l})$ and protective $(327.6 \pm 12.6 \mathrm{U} / \mathrm{l})$ studies, the plasma ALT levels of the test control were significantly $(P<0.05)$ higher than those of all the other groups. The CALE- $75 \mathrm{mg}$ group had the lowest plasma ALT level $(115.8 \pm 4.7 \mathrm{U} / 1)$ in the ameliorative study, 
while the PTSE-50 mg group had the lowest value $(136.3 \pm 5.7 \mathrm{U} / \mathrm{l})$ in the protective study.

\section{Percentage recovery and protection}

The administration of the extracts and metformin prevented (in the protective study) or attenuated (in the ameliorative study) doxorubicin-induced increase in plasma markers of kidney and liver function/integrity and afforded subsequent protection or recovery towards near-normal values. These findings are presented in Tables 4 and 5 as per cent recovery/protection of the parameters according to the different test treatments. The highest percentage recovery of $427.9 \pm 251.8 \%$ was observed in the urea nitrogen/creatinine ratio of the PTSE-50 mg group, while the lowest recovery of $14.9 \pm 4.2 \%$ was observed in the plasma AST level of the CALE-100 mg group. The highest percentage protection of $378.3 \pm 53.1 \%$ was observed in the urea nitrogen/creatinine ratio of the CALE-100 mg group, while the lowest protection of $10.3 \pm 13.8 \%$ was observed in the CALE-100 mg group.

\section{Discussion}

Plasma ALT, AST, ALP, and total bilirubin are the usual biomarkers for detecting liver damage and liver dysfunction in drug-induced liver injury (Panteghini and Bais, 2015; Ortega-Alonso et al., 2016; European Association for the Study of the Liver, 2019). In the present study, doxorubicin caused significant elevation in plasma levels of ALP, ALT, and AST and decreased plasma albumin, globulin, and total protein contents. This finding is in agreement with the results of other studies which reported doxorubicin-induced elevation in plasma levels of ALT, AST, and ALP and decrease in plasma albumin and total protein concentrations ( $\ddot{O} z$ and İlhan, 2006; Lee et al., 2013; Ahmed et al., 2019a; Ikewuchi et al., 2021b). However, these adverse alterations in plasma levels were prevented or attenuated by treatment with the tested PTSE and CALE extracts to varying degrees, as shown by the values of their percentage recovery and protection in Tables 4 and 5 . As shown in both tables, the extracts, especially at the doses of $75 \mathrm{mg} / \mathrm{kg}$ and $100 \mathrm{mg} / \mathrm{kg}$ compared favourably with metformin. The lowering of the plasma enzyme markers by the extracts is an indication of their hepatoprotective potential (Ikewuchi et al., 2017). The extracts may have protected the hepatic cell membrane from doxorubicin-induced damage, thereby restricting the leakage of the enzymes from the hepatocytes into the plasma (Ikewuchi et al., 2021b). This hepatoprotective effect is in corroboration with earlier reports of the hepatoprotective effects of the extracts of $P$. tuberregium sclerotia in alloxan-induced diabetic rabbits (Ikewuchi et al., 2017) and salt-induced hypertensive rats (Ikewuchi et al., 2013a) and of $C$. aconitifolius leaves on liver damage induced by ethanol (Adaramoye et al., 2011), paracetamol (Oyagbemi and Odetola, 2010), and carbon tetrachloride (Saba et al., 2010). This hepatoprotective effect of the extracts may be due to the presence of antioxidants (e.g. allicin, caffeic acid, lycopene, catechin, ellagic acid, epicatechin, epigallocatechin gallate, naringenin, quercetin, and silymarin), all of which possess hepatoprotective activities and are known to condition hepatocytes, resulting in enhanced regeneration of parenchymal cells and thereby protection against membrane fragility and leakage of the marker enzymes into the plasma (Awad et al., 2018; Ikewuchi et al., 2021b).

In the present study, doxorubicin increased plasma creatinine and urea levels. This finding agrees with other reports of doxorubicin-induced elevation of plasma creatinine and urea levels ( $\ddot{\mathrm{O}} z$ and İlhan, 2006; Yilmaz et al., 2006; Ikewuchi et al., 2021c). Because of their inverse relationship with glomerular function, plasma biomarkers such as creatinine and urea concentrations are usually monitored to evaluate glomerular function (Crook, 2012; Lamb and Price, 2015; Meisenberg and Simmons, 2017; Lieberman and Peet, 2018; Ikewuchi et al., 2021c). Therefore, the reduction in plasma creatinine and urea levels by the extracts is suggestive of their capacity to protect nephrons from doxorubicin-induced damage (Jambhulkar et al., 2014) and thus preserve the functional capacity of the glomerular filtration system (Ikewuchi et al., 2021c). The lowering of plasma creatinine, urea, and blood urea nitrogen levels by the extracts may be due to their content of caffeic and chlorogenic acids (Ikewuchi et al., 2017), both of which are reported to decrease plasma levels of kidney markers to nearnormal levels (Pari and KarthiKesan, 2007; Lou et al., 2016). Similar nephroprotective effects were earlier reported for $P$. tuberregium extract on alloxan-induced diabetic rabbits (Ikewuchi et al., 2017) and on salt-induced hypertensive rats (Ikewuchi et al., 2013a) and for C. aconitifolius extract on carbon tetrachloride (Iwu 
et al., 2020) and ethanol intoxicated (Adaramoye and Aluko, 2011) rats.

\section{Conclusion}

The results of the present study show that the tested extracts of $P$. tuberregium sclerotia and $C$. aconitifolius leaves showed a protective effect against doxorubicininduced hepatorenal toxicity. The results also suggest that the sclerotia of $P$. tuberregium and leaves of $C$. aconitifolius may be potential candidates for the prevention and amelioration of doxorubicin-induced hepatorenal toxicity.

\section{Funding}

This research did not receive any specific grant from funding agencies in the public, commercial, or not-for-profit sectors.

\section{References}

Achi N.K., Ohaeri O.C., Ijeh I.I., Eleazu C. (2017) Modulation of the lipid profile and insulin levels of streptozotocin induced diabetic rats by ethanol extract of Cnidoscolus aconitifolius leaves and some fractions: effect on the oral glucose tolerance of normoglycemic rats. Biomed Pharmacother. 86: 562-569. https://doi.org/10.1016/j.biopha. 2016.11.133

Adaramoye O.A., Aluko A. (2011) Methanolic extract of Cnidoscolus aconitifolius attenuates renal dysfunction induced by chronic ethanol administration in Wistar rats. Alcohol Alcohol. 46(1): 4-9. https://doi.org/10.1093/alcalc/agq082

Adaramoye O.A., Aluko A., Oyagbemi A.A. (2011) Cnidoscolus aconitifolius leaf extract protects against hepatic damage induced by chronic ethanol administration in Wistar rats. Alcohol Alcohol. 46(4): 451-458. https://doi.org/10.1093/ alcalc/agr060

Afsar T., Razak S., Batoo K.M., Khan M.R. (2017) Acacia hydaspica R. Parker prevents doxorubicin-induced cardiac injury by attenuation of oxidative stress and structural cardiomyocyte alterations in rats. BMC Complement. Altern. Med. 17(1): 554. https://doi.org/10.1186/s12906-017-2061-0

Ahmed O.M., Abdul-Hamid M.M., El-Bakry A.M., Mohammed H.M., Abdel Rahman F.E.Z.S. (2019a) Camellia sinensis and epicatechin abate doxorubicin-induced hepatotoxicity in male Wistar rats via their modulatory effects on oxidative stress, inflammation, and apoptosis. J. Appl. Pharm. Sci. 9(04): 030-044. https://doi.org/10.7324/JAPS.2019. 90405

Ahmed O.M., Abdul-Hamid M.M., El-Bakry A.M., Mohammed H.M., Abdel Rahman F.E.Z.S. (2019b) Effects of green tea infusion and epicatechin on doxorubicin-induced renocardiotoxicity in male albino rats. Int. J. Pharm. Sci. Res. 10(5): 1000-1014.

Alghorabi A.A., Kabel A.M., Abd Elmaaboud M.A. (2019) Doxorubicin: insights into dynamics, clinical uses and adverse effects. J. Cancer Res. Treat. 7(1): 17-20. https:// doi.org/10.12691/jcrt-7-1-3

Awad N.E., Kassem H.A., Hamed M.A., El-Feky A.M., El-Naggar M. (2018) Hepatoprotective evaluation and isolation of the major secondary metabolites from the ethyl acetate extract of liquid culture filtrate of Chaetomium globosum. Biomed. Pharmacother. 97: 174-180. https://doi.org/ 10.1016/j.biopha.2017.10.120

Azeez O.I., Oyagbemi A.A., Oyeyemi M.O., Odetola A.A. (2010) Ameliorative effects of Cnidoscolus aconitifolius on alloxan toxicity in Wistar rats. Afr. Health Sci. 10(3): 283-291.

Bordbar M., Saleh F., Zekavat O.R., Basiratnia M., Fathpour G., Zareifar S., Shahriari M., Karimi M., Shakibazad N. (2019) Deferoxamine protective effect in preventing nephrotoxicity in children under treatment with doxorubicin: a randomized clinical trial. Iran. J. Blood Cancer 11(2): 51-56.

Carvalho C., Santos R.X., Cardoso S., Correia S., Oliveira P.J., Santos M.S., Moreira P.I. (2009) Doxorubicin: the good, the bad and the ugly effect. Curr. Med. Chem. 16(25): 3267-3285.https://doi.org/10.2174/092986780978880 3312

Chen X., Zhang Y., Zhu Z., Liu H., Guo H., Xiong C., Xie K., Zhang X., Su S. (2016) Protective effect of berberine on doxorubicin-induced acute hepatorenal toxicity in rats. Mol. Med. Rep. 13: 3953-3960. https://doi.org/10.3892/ $\mathrm{mmr} .2016 .5017$

Crook M.A. (2012) Clinical biochemistry and metabolic medicine. London: Hodder and Stoughton Ltd.

El-Shitany N.A., El-Haggar S., El-Desoky K. (2008) Silymarin prevents adriamycin-induced cardiotoxicity and nephrotoxicity in rats. Food Chem. Toxicol. 46(7): 2422-2428. https://doi.org/10.1016/j.fct.2008.03.033

European Association for the Study of the Liver (2019) EASL Clinical Practice Guidelines: drug-induced liver injury. J. Hepatol. 70: 1222-1261.

FAO (1991) Protein quality evaluation: report of Joint FAO/WHO Expert Consultation. FAO Food and Nutrition Paper 51. Rome: Food and Agriculture Organization of the United Nations

Gokcimen A., Cim A., Tola H.T., Bayram D., Kocak A., Özgüner F., Ayata A. (2007) Protective effect of $N$-acetylcysteine, caffeic acid and vitamin $E$ on doxorubicin hepatotoxicity. Hum. Exp. Toxicol. 26(6): 519-525. https://doi. org/10.1177/0960327107076885

Ifeanacho M.O., Ikewuchi C.C., Ikewuchi J.C. (2019a) Antidiabetic effect of a flavonoid and sitosterol - rich aqueous extract of Pleurotus tuberregium sclerotia in alloxaninduced diabetic rabbits. Endocr. Metab. Immune Disord. Drug Targets 19(8): 1148-1156. https://doi.org/10.2174/ 1871530319666190206213843

Ifeanacho M.O., Ikewuchi C.C., Ikewuchi J.C. (2019b) $\mathrm{Nu}$ trient and bioactive phytochemical compositions of Cnidoscolus aconitifolius. Malaysian J. Biochem. Mol. Biol. 22(2): $26-36$.

Ifeanacho M.O., Ikewuchi C.C., Ikewuchi J.C. (2020) Modulation of hematological indices of normal and alloxan-induced dia- 
betic rabbits by aqueous extract of Pleurotus tuberregium sclerotia. Endocr. Metab. Immune Disord. Drug Targets 20(3): 380-387. https://doi.org/10.2174/ 1871530319666 190809155506

Ijeh I.I., Okwujiako I.A., Nwosu P.C., Nnodim H.I. (2009) Phytochemical composition of Pleurotus tuberregium and effect of its dietary incorporation on body/organ weights and serum triacylglycerols in albino mice. J. Med. Plants Res. 3(11): 939-943.

Ikewuchi J.C., Ikewuchi C.C., Ifeanacho M.O., Jaja V.S., Okezue E.C., Jamabo C.N., Adeku K.A. (2021a) Attenuation of doxorubicin-induced cardiotoxicity in Wistar rats by aqueous leaf-extracts of Chromolaena odorata and Tridax procumbens. J. Ethnopharmacol. 274: 114004. https:// doi.org/10.1016/j.jep.2021.114004

Ikewuchi C.C., Ikewuchi J.C., Ifeanacho M.O., Jack D.P., Ikpe C.N., Ehiosun S., Ajayi T.B. (2021b) Protective effect of aqueous leaf extracts of Chromolaena odorata and Tridax procumbens on doxorubicin-induced hepatotoxicity in Wistar rats. Porto Biomed. J. 6: e143. https://doi.org/ 10.1097/j.pbj.0000000000000143

Ikewuchi C.C., Ifeanacho M.O., Ikewuchi J.C. (2021c) Moderation of doxorubicin-induced nephrotoxicity in Wistar rats by aqueous leaf-extracts of Chromolaena odorata and Tridax procumbens. Porto Biomed. J. 6: e129. https://doi.org/ 10.1097/j.pbj.0000000000000129

Ikewuchi C.C., Ifeanacho M.O., Ikewuchi J.C. (2021d) Moderation of doxorubicin-induced dyslipidaemia in Wistar rats by aqueous extracts of Pleurotus tuberregium sclerotia and Cnidoscolus aconitifolius leaves. Malaysian J. Biochem. Mol. Biol. (in press).

Ikewuchi C.C., Ikewuchi J.C. (2009) Chemical profile of Pleurotus tuberregium (Fr) Sing's sclerotia. Pac. J. Sci. Technol. 10(1): 357-362.

Ikewuchi C.C., Ikewuchi J.C., Ifeanacho M.O. (2017) Restoration of plasma markers of liver and kidney functions/integrity in alloxan-induced diabetic rabbits by aqueous extract of Pleurotus tuberregium sclerotia. Biomed. Pharmacother. 95: 1809-1814. https://doi.org/10.1016/j.biopha. 2017.09.075

Ikewuchi J.C, Ikewuchi C.C. (2011) Nutrient composition of Pleurotus tuberregium ( $F r$ ) Sing's sclerotia. Global J. Pure Appl. Sci. 17(1): 51-54.

Ikewuchi J.C., Ikewuchi C.C., Ifeanacho M.O., Igboh N.M. (2014) Blood pressure lowering activity of a flavonoid and phytosterol rich extract of the sclerotia of Pleurotus tuberregium (Fr) Sing's in salt-loaded rats. Biomed. Prevent. Nutr. 4(2): 257-263. https://doi.org/10.1016/j.bionut. 2014.02.006

Ikewuchi J.C., Ikewuchi C.C., Ifeanacho M.O., Igboh N.M., Ijeh I.I. (2013a) Moderation of hematological and plasma biochemical indices of sub-chronic salt-loaded rats by aqueous extract of the sclerotia of Pleurotus tuberregium (Fr) Sing's: Implications for the reduction of cardiovascular risk. J. Ethnopharmacol. 150(2): 466-476. https://doi.org/ 10.1016/j.jep.2013.09.002
Ikewuchi J.C., Ikewuchi C.C., Ifeanacho M.O., Igboh N.M., Ijeh I.I. (2013b) Gas chromatography-flame ionization detector analysis of the phytochemical composition of Pleurotus tuberregium (Fr) Sing's sclerotia: potential benefits. Pac. J. Sci. Technol. 14(2): 342-359.

Indu R., Azhar T.S., Nair A., Nair C.K. (2014) Amelioration of doxorubicin induced cardio-and hepato-toxicity by carotenoids. J. Cancer Res. Ther. 10(1): 62-67. https://doi.org/ 10.4103/0973-1482.131370

Iwu C.C., Akwukwaegbu P.I., Ikewuchi C.C. (2020) Effect of Cnidoscolus aconitifolius leaf extract on selected renal parameters and hematological indices of carbon tetrachloride induced toxic rats. Int. J. Biochem. Biophys. 8(1): 1-8. https://doi.org/10.13189/ijbb.2020.080101

Jagetia G.C., Lalrinpuii T. (2018) Naringin protects rat lung against the doxorubicin-induced biochemical injury. MOJ Anat. Physiol. 5(2): 134-140.

Jambhulkar S., Deshireddy S., Jestadi D.B., Periyasamy L. (2014) Quercetin attenuating doxorubicin induced hepatic, cardiac and renal toxicity in male albino Wistar rats. Amer. J. Phytomed. Clin. Therapeut. 2(8): 985-1004.

Kalender Y., Yel M., Kalender S. (2005) Doxorubicin hepatotoxicity and hepatic free radical metabolism in rats. The effects of vitamin $E$ and catechin. Toxicology 209(1): 39-45. https://doi.org/10.1016/j.tox.2004.12.003

Kuri-García A., Chávez-Servín J.L., Guzmán-Maldonado S.H. (2017) Phenolic profile and antioxidant capacity of Cnidoscolus chayamansa and Cnidoscolus aconitifolius: a review. J. Med. Plants Res. 11(45): 713-727. https://doi.org/ 10.5897/JMPR2017.6512

Lamb E.J., Price C.P. (2015) Kidney function tests - creatinine, urea, and uric acid. [in:] Tietz fundamentals of clinical chemistry and molecular diagnostics. Ed. Burtis C.A., Bruns D.E. St. Louis, Missouri. Saunders: 364-375.

Lee I.C., Kim S.H., Baek H.S., Moon C., Bae C.S., Kim S.H., Yun W.K., Nam K.H., Kim H.C., Kim J.C. (2013) Melatonin improves adriamycin-induced hepatic oxidative damage in rats. Mol. Cell. Toxicol. 9: 257-265. https://doi.org/ 10.1007/s13273-013-0033-0

Lieberman M., Peet A. (2018) Marks' basic medical biochemistry - a clinical approach, fifth edition. Philadelphia. Wolters Kluwer.

Lou J., Gu X., Xing Y., Cui J., Lv W., Zhang Y. (2016) Chlorogenic acid slows down proteinuria and renal fibrosis in 5/6-nephrectomized rats by anti-oxidation and inhibiting accumulation of extracellular matrix. Int. J. Clin. Exp. Med. 9: 15719-15727.

Manoeuvrier G., Bach-Ngohou K., Batard E., Masson D., Trewick D. (2017) Diagnostic performance of serum blood urea nitrogen to creatinine ratio for distinguishing prerenal from intrinsic acute kidney injury in the emergency department. BMC Nephrology 18: 173. https://doi.org/ 10.1186/s12882-017-0591-9

Meisenberg G., Simmons W.H. (2017) Principles of medical biochemistry. Philadelphia, PA: Elsevier, Inc.

National Research Council (2011) Guide for the care and use of laboratory animals. Washington, DC: The National Academies Press. https://doi.org/10.17226/12910. 
Octavia Y., Tocchetti C.G., Gabrielson K.L., Janssens S., Crijns H.J., Moens A.L. (2012) Doxorubicin-induced cardiomyopathy: From molecular mechanisms to therapeutic strategies. J. Mol. Cell. Cardiol. 52(6): 1213-1225. https:// doi.org/10.1016/j.yjmcc.2012.03.006

Oladeinde F.O., Kinyua A.M., Laditan A.A., Michelin R., Bryant J.L., Denaro F., Makinde J.M., Williams A.L., Kennedy A.P., Bronner Y. (2007) Effect of Cnidoscolus aconitifolius leaf extract on the blood glucose and insulin levels of inbred type 2 diabetic mice. Cell. Mol. Biol. 53(3): 68-74. https://doi.org/10.1170/T794

Omar W.M., Raslan Y.A., Amany A.E.A., Eman M.R, Gehan S.G. (2016) The ameliorative effect of ellagic acid and rosemarinic acid against cardio-nephrotoxicity induced by doxorubicin in rats. Int. J. Sci. Res. Pub. 6(2): 249-256.

Onasanwo S.A., Adebayo O.G., Nicodemus O.U., Ajayi A.M., Wadioni A., Wopara I. (2020) Hematoprotective and gastroprotective effects of Cnidoscolus aconitifolius (Linn)supplemented diet in male Wistar rats. Ann. Res. Rev. Biol. 35(2): 107-118. https://doi.org/10.9734/ARRB/ 2020/v35i230194

Ortega-Alonso A., Stephens C., Lucena M.I., Andrade R.J. (2016) Case characterisation, clinical features and risk factors in drug-induced liver injury. Int. J. Mol. Sci. 17: e714.

Otitolaiye C.A., Asokan C. (2016) GC-MS Analysis of Cnidoscolus aconitifolius leaf aqueous extracts. Int. J. Sci. Res. 5(8): 471-475. https://doi.org/10.21275/ART2016727

Oyagbemi A.A., Odetola A.A. (2010) Hepatoprotective effects of ethanolic extract of Cnidoscolus aconitifolius on paracetamol-induced hepatic damage in rats. Pak. J. Biol. Sci. 13(4): 164-169. https://doi.org/10.3923/pjbs.2010.164. 169

Öz E., Ilhan M.N. (2006) Effects of melatonin in reducing the toxic effects of doxorubicin. Mol. Cell. Biochem. 286: 11-15. https://doi.org/10.1007/s11010-005-9003-8

Panteghini M., Bais R. (2015) Serum enzymes. [in:] Tietz fundamentals of clinical chemistry and molecular diagnostics. Ed. Burtis C.A., Bruns D.E. St. Louis. Missouri. Saunders: 319-336.

Pari L., KarthiKesan K. (2007) Protective role of caffeic acid against alcohol-induced biochemical changes in rats. Fundam. Clin. Pharmacol. 21: 355-361. https://doi.org/ 10.1111/j.1472-8206.2007.00505.x

Patel N., Joseph C., Corcoran G.B., Ray S.D. (2010) Silymarin modulates doxorubicin-induced oxidative stress, Bcl-xL and p53 expression while preventing apoptotic and necrotic cell death in the liver. Toxicol. Appl. Pharmacol. 245(2): 143-152. https://doi.org/10.1016/j.taap.2010.02.002

Paul S. (2013) Biochemical test profiles. [in:] Medical laboratory technology, vol. III: A procedure manual for routine diagnostic tests,. Ed. Mukherjee K.L, Gosh S. New Delhi. Tata McGraw-Hill Education: 952-970.

Rashid S., Ali N., Nafees S., Ahmad S.T., Arjumand W., Hasan S.K., Sultana S. (2013) Alleviation of doxorubicin-induced nephrotoxicity and hepatotoxicity by chrysin in Wistar rats. Toxicol. Mech. Methods 23(5): 337-345. https://doi.org/ $10.3109 / 15376516.2012 .759306$
Rašković A., Stilinović N., Kolarović J., Vasović V., Vukmirović S, Mikov M. (2011) The protective effects of silymarin against doxorubicin-induced cardiotoxicity and hepatotoxicity in rats. Molecules 16: 8601-8613. https://doi.org/ 10.3390/molecules 16108601

Ren X., Bo Y., Fan J., Chen M., Xu D., Dong Y., He H., Ren X., Qu R., Jin Y., Zhao W., Xu C. (2016) Dalbergioidin ameliorates doxorubicin-induced renal fibrosis by suppressing the TGF $\beta$ signal pathway. Mediat. Inflam. 2016: 5147571. https://doi.org/10.1155/2016/5147571

Rudolfová P., Hanušová V., Skálová L., Bártíková H., Matoušková P., Boušová, I. (2014) Effect of selected catechins on doxorubicin antiproliferative efficacy and hepatotoxicity in vitro. Acta Pharm. 64(2): 199-209. https://doi.org/ 10.2478/acph-2014-0018

Saba A.B., Oyagbemi A.A., Azeez, O.I. (2010) Amelioration of carbon tetrachloride-induced hepatotoxicity and haemotoxicity by aqueous leaf extract of Cnidoscolus aconitifolius in rats. Niger. J. Physiol. Sci. 25(2): 139-147.

Sabapathy V., Cheru N.T., Corey R., Mohammad S., Sharma R. (2019) A novel hybrid cytokine IL233 mediates regeneration following doxorubicin-induced nephrotoxic injury. Sci. Rep. 9(1): 3215. https://doi.org/10.1038/s41598-01939886-9

Song S., Chu L., Liang H., Chen J., Liang J., Huang Z., Zhang B., Chen X. (2019) Protective effects of dioscin against doxorubicin-induced hepatotoxicity via regulation of Sirt1/ FOXO1/NF b bignal. Front. Pharmacol. 10: 1030. https:// doi.org/10.3389/fphar.2019.01030

Surai P.F. (2015) Silymarin as a natural antioxidant: an overview of the current evidence and perspectives. Antioxidants 4: 204-247. https://doi.org/10.3390/antiox4010204

Vapa I., Torres V.M., Djordjevic A., Vasovic V., Srdjenovic B., Simic V.D., Popović J.K. (2012) Effect of fullerenol $\mathrm{C}_{60}(\mathrm{OH})_{24}$ on lipid peroxidation of kidneys, testes and lungs in rats treated with doxorubicin. Eur. J. Drug Metab. Pharmacokinet. 37(4): 301-307. https://doi.org/10.1007/ s13318-012-0092-y

Yagmurca M., Erdogan H., Iraz M., Songur A., Ucar M., Fadillioglu E. (2004) Caffeic acid phenethyl ester as a protective agent against doxorubicin nephrotoxicity in rats. Clin. Chim. Acta 348(1-2): 27-34. https://doi.org/10.1016/ j.cccn.2004.03.035

Yilmaz S., Atessahin A., Sahna E., Karahan I, Ozer S. (2006) Protective effect of lycopene on Adriamycin-induced cardiotoxicity and nephrotoxicity. Toxicology 218(2-3): 164-171. https://doi.org/10.1016/j.tox.2005.10.015

Zilinyi R., Czompa A., Czegledi A., Gajtko A., Pituk D., Lekli I., Tosaki A. (2018) The cardioprotective effect of metformin in doxorubicin-induced cardiotoxicity: the role of autophagy. Molecules 23: 1184. https://doi.org/10.3390/ molecules23051184 